\title{
Cosmic acceleration in a model of scalar-tensor gravitation
}

\author{
Sanil Unnikrishnan ${ }^{\dagger}$ and T. R. Seshadri $\ddagger$ \\ Department of Physics 83 Astrophysics, \\ University of Delhi, Delhi 11000\%, India. \\ †e-mail : sanil@physics.du.ac.in \\ †e-mail : trs@physics.du.ac.in
}

October 27, 2018

\begin{abstract}
In this paper we consider a model of scalar-tensor theory of gravitation in which the scalar field, $\phi$ determines the gravitational coupling $\mathrm{G}$ and has a Lagrangian of the form, $\mathcal{L}_{\phi}=-V(\phi) \sqrt{1-\partial_{\mu} \phi \partial^{\mu} \phi}$. We study the cosmological consequence of this theory in the matter dominated era and show that this leads to a transition from an initial decelerated expansion to an accelerated expansion phase at the present epoch. Using observational constraints, we see that the effective equation of state today for the scalar field turns out to be $p_{\phi}=w_{\phi} \rho_{\phi}$, with $w_{\phi}=-0.88$ and that the transition to an accelerated phase happened at a redshift of about 0.3 .
\end{abstract}

\section{Introduction}

Various observations strongly indicate that at the present epoch the universe is in a phase of accelerated expansion [1, 2]. This accelerated expansion of the universe might be caused by a non-zero value of the cosmological constant [3, 4, 5], or an evolving scalar field such as quintessence [6, 7], phantom field 
[8, 9, 10, 11], tachyon field [12, 13, 14] etc. For a detailed review of dark energy see for example Ref.[15].

Quintessence refers to a scalar field whose Lagrangian is of the form

$$
\mathcal{L}_{\phi}=\frac{1}{2} \partial_{\mu} \phi \partial^{\mu} \phi-V(\phi)
$$

In this paper we investigate the consequences of a scalar field whose Lagrangian is given by,

$$
\mathcal{L}_{\phi}=-V(\phi) \sqrt{1-\partial_{\mu} \phi \partial^{\mu} \phi}
$$

Without going into the details of the fundamental origin of this form, we use this form of the action at a phenomenological level. It is, however, appropriate to mention that this form of the Lagrangian arises from string theory. A detailed investigation of this form of Lagrangian in the context of Einstein-Hilbert action has been studied in Ref[14. Quite independently (as described in [16]) the structure of this Lagrangian can also be motivated from the fact that its form is very similar to that of a relativistic particle in classical mechanics $\left(\mathcal{L}=-m \sqrt{1-\dot{q}^{2}}\right)$.

Most of these scalar field models of Dark energy are discussed in the context of Einstein-Hilbert action in general relativity. A well known alternative to Einstein's theory is the Brans Dicke theory [17] where Newton's gravitational constant $G$ is identified with the inverse of a scalar field whose dynamics is governed by the matter distribution. The Brans Dicke theory is motivated from Mach's Principle which in a way suggest that $G$ should be function of epoch [17, 18, 19. Brans Dicke theory can be considered as a special case of more general scalar tensor theory of gravitation. Accelerated expansion of the universe in scalar tensor theories of gravitation has been studied by various authors [20, 21, 22, 23, 24, 25, 26, 27]. These authors, however, considered a scalar field Lagrangian which has a standard form $\mathcal{L}_{\phi}=\frac{1}{2} \partial_{\mu} \phi \partial^{\mu} \phi-V(\phi)$. In this paper we consider a scalar field which plays a role similar to the Brans-Dicke field but with a Lagrangian of the form $\mathcal{L}_{\phi}=-V(\phi) \sqrt{1-\partial_{\mu} \phi \partial^{\mu} \phi}$.

Throughout this paper we use the units $\hbar=c=1$. We consider the case when $G=\phi^{2}$ and $V(\phi)=\lambda \phi^{-4}$ where $\lambda$ is a dimensionless parameter (in the units $\hbar=c=1$ ). As pointed out earlier, although this form of the action arises in certain models of string theory [28, 29], we use this action at a purely phenomenological level, without analyzing the details of the fundamental origin of this form of the action. 
The precise form of the action and the resulting field equations are given in section 2. We discuss in section 3 the cosmological consequences of this theory in the matter dominated era and show that this leads to an accelerated expansion of the universe at the present epoch. The conclusions of this analysis are summarized in section 4.

\section{Action and the field equations}

In this paper we consider the action for the scalar tensor gravitation of the form:

$$
\mathcal{A}=\frac{1}{16 \pi} \int\left[-\frac{R}{\phi^{2}}-\frac{\lambda}{\phi^{4}} \sqrt{1-\partial_{\mu} \phi \partial^{\mu} \phi}+16 \pi \mathcal{L}_{m}\right] \sqrt{-g} d^{4} x
$$

We may note the following features of this action. As pointed out earlier, the scalar field $\phi$ plays a role similar to the Brans-Dicke field $\phi_{B D}$ in the standard Brans-Dicke theory. In the latter, the value of $G$ is determined by $\phi_{B D}^{-1}$. In our model the value of $G$ is determined by $\phi^{2}$. Further, the 'kinetic energy' part of $\phi$ in the second term in the action, is of a form different from the standard Brans-Dicke case. The function $V(\phi)$ has the dimensions of energy density and in the units we use here, $(\hbar=c=1)$, it has the units of $M^{4}$. Further, in these units $\phi$ has dimensions of $M^{-1}$. With these facts in mind, we consider a simple form of $V(\phi)$ given by,

$$
V(\phi)=\lambda \phi^{-4}
$$

where, $\lambda$ is a dimensionless parameter. Varying the action given in Eq. (3), the Einstein's equation and the equation of motion for the field are:

$$
\begin{aligned}
G_{a b}= & 8 \pi \phi^{2} T_{a b}-\frac{2}{\phi}\left[\phi_{a ; b}-g_{a b} \square \phi\right]+\frac{6}{\phi^{2}}\left[\phi_{a} \phi_{b}-g_{a b} \phi_{\mu} \phi^{\mu}\right] \\
& +\frac{\lambda}{2 \phi^{2}}\left[\frac{\phi_{a} \phi_{b}}{\sqrt{1-\phi_{\mu} \phi^{\mu}}}+g_{a b} \sqrt{1-\phi_{\mu} \phi^{\mu}}\right] \\
R= & \frac{2 \lambda}{\phi^{2}} \frac{1}{\sqrt{1-\phi_{\mu} \phi^{\mu}}}+\frac{\lambda \square \phi}{2 \phi \sqrt{1-\phi_{\mu} \phi^{\mu}}}+\frac{\lambda \phi^{\mu}\left(\phi_{i} \phi^{i}\right)_{\mu}}{4 \phi\left(1-\phi_{\mu} \phi^{\mu}\right)^{3 / 2}}
\end{aligned}
$$

where $T_{a b}$ is the energy momentum tensor of the matter. $T_{a b}$ is defined as :

$$
T^{a b}=-\frac{1}{2 \sqrt{-g}} \frac{\delta}{\delta g_{a b}}\left(\mathcal{L}_{m} \sqrt{-g}\right)
$$




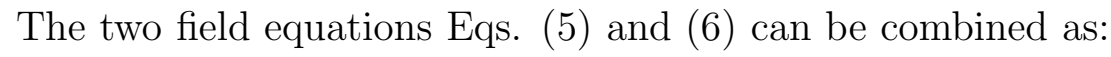

$$
\begin{aligned}
8 \pi \phi^{2} T= & -\frac{6 \square \phi}{\phi}+\frac{18 \phi_{\mu} \phi^{\mu}}{\phi^{2}}+\frac{3 \lambda}{2 \phi^{2}} \frac{\phi_{\mu} \phi^{\mu}}{\sqrt{1-\phi_{\mu} \phi^{\mu}}} \\
& -\frac{\lambda}{2 \phi} \frac{\square \phi}{\sqrt{1-\phi_{\mu} \phi^{\mu}}}-\frac{\lambda}{4 \phi} \frac{\phi^{\mu}\left(\phi_{a} \phi^{a}\right)_{, \mu}}{\left(1-\phi_{\mu} \phi^{\mu}\right)^{3 / 2}}
\end{aligned}
$$

This equation [Eq. (8)] shows that the evolution of $\phi$ is determined by matter distribution (through its trace $T$ ) just as in Brans Dicke Theory but here the equation is not in as simple form as in Brans Dicke Theory.

In this theory we have one dimensionless parameter $\lambda$ whose value must be fixed observationally. We will fix its value by considering the cosmological evolution determined by this theory and comparing it with the observed parameters for the universe.

\section{Cosmological Consequences}

The field $\phi$, in general, will be a function of space and time i.e $\phi=\phi(\vec{x}, t)$. We, however, will consider the field to be homogeneous and isotropic on cosmic scales. Hence, the field $\phi=\phi(t)$. We will consider spatially flat Friedmann Robertson Walker metric of the form:

$$
d s^{2}=d t^{2}-a(t)^{2}\left[d r^{2}+r^{2} d \theta^{2}+r^{2} \sin \theta^{2} d \phi^{2}\right]
$$

Here $a(t)$ is the scale factor. The Friedmann equations governing the evolution of $a(t)$ are given by

$$
\begin{aligned}
\left(\frac{\dot{a}}{a}\right)^{2}= & \frac{8 \pi \phi^{2}}{3} \rho+2 \frac{\dot{\phi} \dot{a}}{\phi a}+\frac{\lambda}{6 \phi^{2} \sqrt{1-\dot{\phi}^{2}}} \\
\left(\frac{\ddot{a}}{a}\right)= & -\frac{4 \pi \phi^{2}}{3}(\rho+3 p)+\frac{\dot{\phi} \dot{a}}{\phi a}+\frac{\ddot{\phi}}{\phi}-3 \frac{\dot{\phi}^{2}}{\phi^{2}} \\
& +\frac{\lambda}{4 \phi^{2}} \sqrt{1-\dot{\phi}^{2}}-\frac{\lambda}{12 \phi^{2}} \frac{1}{\sqrt{1-\dot{\phi}^{2}}}
\end{aligned}
$$

With $\phi=\phi(t)$ the equation for $\phi($ Eq.(8) $)$ takes the form: 


$$
\begin{aligned}
8 \pi \phi^{2}(\rho-3 p)= & -6 \frac{\ddot{\phi}}{\phi}-18 \frac{\dot{\phi} \dot{a}}{\phi a}+18 \frac{\dot{\phi}^{2}}{\phi^{2}}+\frac{3 \lambda \dot{\phi}^{2}}{2 \phi^{2} \sqrt{1-\dot{\phi}^{2}}} \\
& -\frac{\lambda \ddot{\phi}}{2 \phi \sqrt{1-\dot{\phi}^{2}}}-\frac{3 \lambda \dot{a} \dot{\phi}}{2 a \phi \sqrt{1-\dot{\phi}^{2}}}-\frac{\lambda \dot{\phi}^{2} \ddot{\phi}}{2 \phi\left(1-\dot{\phi}^{2}\right)^{3 / 2}}
\end{aligned}
$$

Here $\rho=T_{0}^{0}$ and $p=-T_{1}^{1}=-T_{1}^{1}=-T_{1}^{1}$ which is the energy density and the pressure, respectively.

In our model we assume the universe to consist of the pressureless matter in addition to the field $\phi$. The contribution from radiation at late time (as is the case for the present era) can be neglected. Hence, we drop the contribution of radiation in the source term for Einstein's equation. In addition to equations (10) and (11) we also have the continuity equation for matter.

$$
\dot{\rho}=-3 H(\rho+p)
$$

For pressure less matter this gives:

$$
\rho_{m}(a)=\rho_{m 0} \frac{a_{0}^{3}}{a^{3}}
$$

where $\rho_{m 0}$ is the matter density at the present epoch and $a_{0}$ is the scale factor at the present epoch.

In order to solve these equations (Eqs.(10) and (11)) we need the values of $\rho_{m 0}$ and $\lambda$ as well as the values of $a, \phi$ and $\dot{\phi}$ at some instant of time. We now fix these values from the observations at the present epoch. First of all we normalize the scale factor to unity at the present epoch. At the present epoch $\dot{a}\left(t_{0}\right)=H_{0}\left(\right.$ as $\left.a\left(t_{0}\right)=1\right)$ which is the Hubble constant. The value of $\phi$ at the present epoch will be fixed as $\phi\left(t_{0}\right)=\sqrt{G_{0}}$ where $G_{0}$ is the Newtonian gravitational constant at the present epoch. There is strong evidence from both experiments as well as cosmological observation that there is no significant variation of the gravitational constant at the present epoch. These considerations will be consistent if we consider the simplest case when $\dot{\phi}\left(t_{0}\right)=0$ which ensures that $\dot{G}\left(t_{0}\right)=0$. So we consider the following conditions at the present epoch, $t=t_{0}$ :

$$
\begin{aligned}
& a\left(t_{0}\right)=1 \\
& \dot{a}\left(t_{0}\right)=H_{0}
\end{aligned}
$$




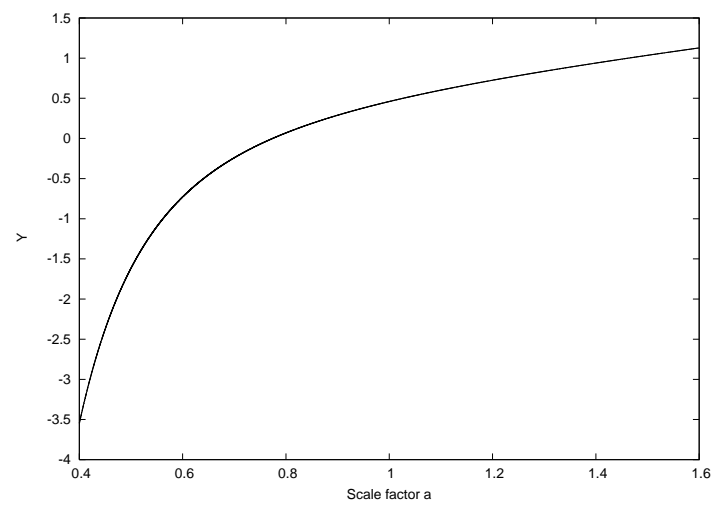

Figure 1: This plot shows how $\ddot{a}$ evolves with scale factor. Here $Y=\ddot{a} / H_{0}^{2}$

$$
\begin{aligned}
& \phi\left(t_{0}\right)=\sqrt{G_{0}} \\
& \dot{\phi}\left(t_{0}\right)=0
\end{aligned}
$$

With these conditions we can determine the value of $\lambda$ from Eq. (10). On substituting Eqs. (15) to (18) in the Friedmann equation (10) we get:

$$
\lambda=6 G_{0} H_{0}^{2}\left(1-\Omega_{m}\right),
$$

where $\Omega_{m}$ is the density parameter of (pressureless) matter today and is defined as

$$
\Omega_{m}=\frac{8 \pi G_{0} \rho_{m 0}}{3 H_{0}^{2}}
$$

Observations indicate that $\Omega_{m}=0.27$ and $H_{0}=70 \mathrm{~km} / \mathrm{s} / \mathrm{Mpc}$ (as indicated by the observations by WMAP [30]). In the units of $\hbar=c=1$ this gives the value $H_{0}=6.1207 \times 10^{-61} M_{p}$ and with the value of Newtonian gravitational constant $G_{0}=(8 \pi)^{-1} M_{p}^{-2}$, we obtain the value of $\lambda$ as :

$$
\lambda=6.5281 \times 10^{-122}
$$

Eq.(12) together with conditions Eqs.(15) to (18) gives,

$$
\ddot{\phi}\left(t_{0}\right)=-\frac{6 H_{0}^{2} \Omega_{m} \sqrt{G_{0}}}{(12+\lambda)}
$$

If the time variation of the field $\phi$ is exactly zero in the present epoch it would imply that $\ddot{G}\left(t_{0}\right)$ is negative which in turn implies that $G(t)$ has a 


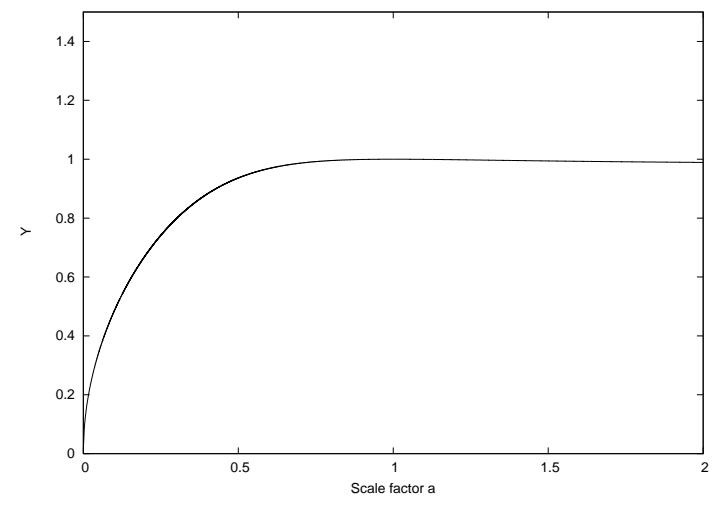

Figure 2: This plot shows how field $\phi$ evolves with scale factor. Here $Y=$ $\phi / \sqrt{G_{0}}$

maxima at the present epoch. Observationally we know that there is no significant variation of the "Gravitational coupling" with time. Hence, with in the framework of this scenario, the present value of the Gravitational coupling parameter should be close to its maximum value. Eq.(11) together with conditions Eqs.(15) to (18) gives,

$$
\frac{\ddot{a}\left(t_{0}\right)}{H_{0}^{2}}=-\frac{\Omega_{m}}{2}+\frac{\ddot{\phi}\left(t_{0}\right)}{H_{0}^{2} \sqrt{G_{0}}}+\frac{\lambda}{6 H_{0}^{2} G_{0}} .
$$

From Eqs. (19) , (22) and (23) we get:

$$
\frac{\ddot{a}\left(t_{0}\right)}{H_{0}^{2}}=1-2 \Omega_{m}=0.46
$$

Hence the conditions Eqs.(15) to (18) naturally leads to an accelerated expansion of the universe at the present epoch. Within the frame work of Einstein GR, if we consider dark energy to be a perfect fluid with equation of state $p_{\phi}=w_{\phi} \rho_{\phi}$, then such a value of $\ddot{a}\left(t_{0}\right)$ (Eq.(24) ) would correspond to $w_{\phi}\left(t_{0}\right)=-0.88$. The figure (11) shows the plot of $\ddot{a} / H_{0}^{2}$ verses the scale factor $a$. We see from the figure that $\ddot{a}$ is less than zero for small values of the scale factor and becomes positive for the large values. The transition occurs for $a=.77$ (corresponding to a redshift of $z=0.3$ ). Thus in this model the transition from a decelerating phase to an accelerating phase should have taken place at $z=0.3$. In figure (2) we plot the ratio $\phi / \sqrt{G_{0}}$ verses the 
scale factor, where $G_{0}$ is the Newtonian gravitational constant. We can see from the figure (Fig.(2) ) that the field $\phi$ asymptotically attains a constant value of $0.98 \times \sqrt{G_{0}}$. A constant value of the field $\phi$ would imply that the field equation (Eq.(5) ) would then become usual Einstein equation with a cosmological constant. Hence this model asymptotically becomes De Sitter universe.

\section{Conclusion and Discussion}

We have considered a model in which the gravitational coupling evolves as $G \sim \phi^{2}$ where the Lagrangian for the scalar field, $\phi$, is given by the Lagrangian of the form $\mathcal{L}_{\phi}=-V(\phi) \sqrt{1-\partial_{\mu} \phi \partial^{\mu} \phi}$. We have chosen a potential of the form $V(\phi)=\lambda \phi^{-4}$, where $\lambda$ is a dimensionless parameter. On considering the cosmological consequence of this theory we have shown that imposing condition that $\dot{\phi}\left(t_{0}\right)=0$ gives a value of $\lambda=6.5281 \times 10^{-122}$ and also gives a positive value of acceleration ( at the present epoch) with $\ddot{a}\left(t_{0}\right)=0.46 H_{0}^{2}$. Such a value of $\ddot{a}$ would correspond to an effective equation of state $w_{\phi}\left(t_{0}\right)=-0.88$. Numerical calculation shows that there was a transition from an initial decelerating expansion to an accelerated expansion phase at the present epoch. This transition occurred at $z=0.3$. This result is consistent with the observations [1, 2]. Hence this could be a possible model for the present observed accelerated expansion of the universe.

\section{Acknowledgment}

S.U. thanks C.S.I.R, India for a Senior Research Fellowship. TRS thanks IUCAA for the support provided through the Associateship Program and DST, India for the project. S.U and TRS thanks IUCAA for the facilities at the IUCAA Reference Centre at Delhi University.

\section{APPENDIX}

\section{Action for scalar tensor theory of gravitation}

Let us assume that the locally measured gravitational constant $G$ is determined by the value of a scalar field $\phi$, i.e $G=f(\phi)$. Action for such a theory would be :

$$
\mathcal{A}=\frac{1}{16 \pi} \int\left[-\frac{R}{f(\phi)}+\mathcal{L}_{\phi}+16 \pi \mathcal{L}_{m}\right] \sqrt{-g} d^{4} x
$$


where $\mathcal{L}_{m}$ is the Lagrangian of matter and $\mathcal{L}_{\phi}$ is the Lagrangian of the scalar field $\phi$ (un-coupled part). Note that in Einstein-Hilbert Action the coefficient of the Ricci scalar $R$ is $1 / 16 \pi G$ but here we have $1 / 16 \pi f(\phi)$. In this paper we have studied the cosmological consequences of a scalar field with the Lagrangian of the form $\mathcal{L}_{\phi}=-V(\phi) \sqrt{1-\partial_{\mu} \phi \partial^{\mu} \phi}$. We now obtain the form of $f(\phi)$ and $V(\phi)$ from the dimensional argument but it will, however, be instructional to first consider briefly the case of a scalar field with the Lagrangian in the standard form, namely, $\mathcal{L}_{\phi}=\frac{1}{2} \partial_{\mu} \phi \partial^{\mu} \phi-V(\phi)$. The dimension of $\phi$ is $M$. Since the dimensions of $G$ as well as $f(\phi)$ is $M^{-2}$. The simplest form for the function $f(\phi)$ would be $f(\phi)=\alpha \phi^{-2}$, where $\alpha$ is a dimensionless parameter. Consider the simplest case when $V(\phi)=0$. With this the action takes the form :

$$
\mathcal{A}=\frac{1}{16 \pi} \int\left[-\frac{\phi^{2} R}{\alpha}+\frac{1}{2} \partial_{\mu} \phi \partial^{\mu} \phi+16 \pi \mathcal{L}_{m}\right] \sqrt{-g} d^{4} x
$$

Defining $\phi_{B D}=\phi^{2} / \alpha$ and $w=\alpha / 8$ the action (26) can be expressed in an alternate form as

$$
\mathcal{A}=\frac{1}{16 \pi} \int\left[-\phi_{B D} R+w \frac{\partial_{\mu} \phi_{B D} \partial^{\mu} \phi_{B D}}{\phi_{B D}}+16 \pi \mathcal{L}_{m}\right] \sqrt{-g} d^{4} x
$$

This is the standard action for the Brans-Dicke Theory with field $\phi_{B D}$ being the Brans Dicke field.

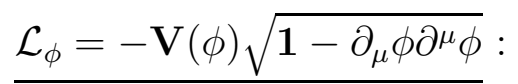

In this case the dimension of scalar field $\phi$ is $M^{-1}$. As the dimension of $f(\phi)$ is $M^{-2}$ the simplest choice from dimensional argument would be $f(\phi)=\phi^{2}$. The dimension of potential $V(\phi)$ is $M^{4}$. So the simplest choice would be to consider $V(\phi)=\lambda \phi^{-4}$, where $\lambda$ is a dimensionless parameter. With this the action (25) becomes

$$
\mathcal{A}=\frac{1}{16 \pi} \int\left[-\frac{R}{\phi^{2}}-\frac{\lambda}{\phi^{4}} \sqrt{1-\partial_{\mu} \phi \partial^{\mu} \phi}+16 \pi \mathcal{L}_{m}\right] \sqrt{-g} d^{4} x
$$

This is the form of action that we have considered in this paper.

[It is interesting to note that the action in Eq.(28) is related to BransDicke action in the limit $\partial_{\mu} \phi \partial^{\mu} \phi<<1$. This limit is reminiscent of the 
non-relativistic limit in particle mechanics where we use $\dot{x} \ll c$. In this limit the action Eq.(28) reduces to the form.

$$
\mathcal{A}=\frac{1}{16 \pi} \int\left[-\frac{R}{\phi^{2}}+\frac{\lambda}{2 \phi^{4}} \partial_{\mu} \phi \partial^{\mu} \phi-\frac{\lambda}{\phi^{4}}+16 \pi \mathcal{L}_{m}\right] \sqrt{-g} d^{4} x
$$

Denoting $\phi_{B D}=\phi^{-2}$ and $w=\lambda / 8$, this action can be expressed in an alternate form

$$
\mathcal{A}=\frac{1}{16 \pi} \int\left[-\phi_{B D} R+w \frac{\partial_{\mu} \phi_{B D} \partial^{\mu} \phi_{B D}}{\phi_{B D}}-8 w \phi_{B D}^{2}+16 \pi \mathcal{L}_{m}\right] \sqrt{-g} d^{4} x
$$

This is the action for Brans-Dicke theory with a quadratic potential for the Brans-Dicke field $\phi_{B D}$. Such a quadratic form of the potential in Brans-Dicke theory has been considered earlier in literature [31].]

\section{References}

[1] A. G. Riess et al., Astron. J. 116, 1009 (1998).

[2] S. Perlmutter et al., Ap. J. 517, 565 (1999).

[3] S. Weinberg, Rev. Mod. Phys. 61, 1 (1989).

[4] T. Padmanabhan, Phys. Report 380, 235 (2003).

[5] P. J. E.Peebles and B. Ratra, Rev. Mod. Phys. 75, 559 (2003).

[6] P. G. Ferreira and M. Joyce, Phys. Rev.D 58, 023503 (1998).

[7] B. Ratra and P. J. E.Peebles, Phys. Rev. D 37, 3406 (1988).

[8] S. M. Carroll, M. Hoffman and M. Trodden, Phys. Rev. D 68, 023509 (2003).

[9] M. Kaplinghat and S. Bridle, Phys. Rev. D 71, 123003 (2005).

[10] R. R. Caldwell, Phys. Lett. B 545, 23 (2002).

[11] M. Sami and A. Toporensky, Mod. Phys. Lett. A 19, 1509 (2004).

[12] T. Padmanabhan, Phys. Rev. D 66, 021301 (2002). 
[13] J. S. Bagla, H. K. Jassal and T. Padmanabhan, Phys. Rev.D 67, 063504 (2003).

[14] E. J. Copeland, M. R. Garousi, M. Sami and S. Tsujikawa, Phys. Rev. D 71, 043003 (2005).

[15] E. J. Copeland, M. Sami and S. Tsujikawa, Int. J. Mod. Phys. D 15, 1753 (2006).

[16] T. Padmanabhan and T. Roy Choudhury, Phys. Rev.D 66, 081301 (2002).

[17] C. Brans and R. H. Dicke, Phys. Rev. 124, 925 (1961).

[18] J. V. Narlikar, An introduction to cosmology, 3rd ed., Cambridge University Press (2002).

[19] Ray D' Inverno, Introducing Einstein's Relativity, Oxford University Press (2002).

[20] L. Perivolaropoulos, JCAP 0510, 001 (2005)

[21] E. Elizalde, S. Nojiri and S. D. Odintsov, Phys. Rev. D 70, 043539 (2004).

[22] L. M. Diaz-Rivera and L. O. Pimentel, Int. J. Mod. Phys A 18, 651 (2003).

[23] A. A. Sen and S. Sen, Mod. Phys. Lett. A 16, 1303 (2001).

[24] G. Esposito-Farese and D. Polarski, Phys. Rev. D 63, 063504 (2001).

[25] B. Boisseau, G. Esposito-Farese, D. Polarski and A. A. Starobinsky, Phys. Rev. Lett. 85, 2236 (2000).

[26] N. Bartolo and M. Pietroni, Phys. Rev. D 61, 023518 (1999).

[27] S. Capozziello, S. Nojiri and S. D. Odintsov, Phys. Lett. B 634, 93 (2006).

[28] Y. S. Piao, Q. G. Huang, X. Zhang and Y. Z. Zhang, Phys. Lett. B 570, 1 (2003). 
[29] P. Chingangbam, S. Panda and A. Deshamukhya, JHEP. 0502, 052 (2005).

[30] C. L. Bennett et al., Ap. J. Suppl. 148, 1 (2003).

[31] O. Bertolami and P. J. Martins, Phys. Rev. D 61, 064007 (2000). 